\title{
The Effect of Noninvasive Telemonitoring for Chronic Heart Failure on Health Care Utilization: Systematic Review
}

Stefan L Auener, MSc; Toine E P Remers, MSc; Simone A van Dulmen, PhD; Gert P Westert, Prof Dr; Rudolf B Kool, MD, PhD; Patrick P T Jeurissen, Prof Dr

IQ healthcare, Radboud Institute for Health Sciences, Radboud University Medical Center, Nijmegen, Netherlands

Corresponding Author:

Stefan L Auener, MSc

IQ healthcare

Radboud Institute for Health Sciences

Radboud University Medical Center

Geert Grooteplein Zuid 10

Nijmegen, 6525GA

Netherlands

Phone: 31243616359

Email: stefan.auener@radboudumc.nl

\section{Abstract}

Background: Chronic heart failure accounts for approximately 1\%-2\% of health care expenditures in most developed countries. These costs are primarily driven by hospitalizations and comorbidities. Telemonitoring has been proposed to reduce the number of hospitalizations and decrease the cost of treatment for patients with heart failure. However, the effects of telemonitoring on health care utilization remain unclear.

Objective: This systematic review aims to study the effect of telemonitoring programs on health care utilization and costs in patients with chronic heart failure. We assess the effect of telemonitoring on hospitalizations, emergency department visits, length of stay, hospital days, nonemergency department visits, and health care costs.

Methods: We searched PubMed, Embase, and Web of Science for randomized controlled trials and nonrandomized studies on noninvasive telemonitoring and health care utilization. We included studies published between January 2010 and August 2020. For each study, we extracted the reported data on the effect of telemonitoring on health care utilization. We used $P<.05$ and CIs not including 1.00 to determine whether the effect was statistically significant.

Results: We included 16 randomized controlled trials and 13 nonrandomized studies. Inclusion criteria, population characteristics, and outcome measures differed among the included studies. Most studies showed no effect of telemonitoring on health care utilization. The number of hospitalizations was significantly reduced in 38\% (9/24) of studies, whereas emergency department visits were reduced in $13 \%$ (1/8) of studies. An increase in nonemergency department visits (6/9, 67\% of studies) was reported. Health care costs showed ambiguous results, with 3 studies reporting an increase in health care costs, 3 studies reporting a reduction, and 4 studies reporting no significant differences. Health care cost reductions were realized through a reduction in hospitalizations, whereas increases were caused by the high costs of the telemonitoring program or increased health care utilization.

Conclusions: Most telemonitoring programs do not show clear effects on health care utilization measures, except for an increase in nonemergency outpatient department visits. This may be an unwarranted side effect rather than a prerequisite for effective telemonitoring. The consequences of telemonitoring on nonemergency outpatient visits should receive more attention from regulators, payers, and providers. This review further demonstrates the high clinical and methodological heterogeneity of telemonitoring programs. This should be taken into account in future meta-analyses aimed at identifying the effective components of telemonitoring programs.

(J Med Internet Res 2021;23(9):e26744) doi: 10.2196/26744

\section{KEYWORDS}

heart failure; telemonitoring; remote monitoring; health care utilization; eHealth 


\section{Introduction}

\section{Background}

Chronic heart failure (CHF) is one of the most prevalent high-cost chronic diseases affecting at least $1 \%-2 \%$ of the worldwide population [1]. Europe and the United States spent approximately $1 \%-2 \%$ of their national health care budget on this chronic disease [1,2]. Worldwide, the economic burden of CHF is estimated to be approximately US $\$ 108$ billion per annum, of which US $\$ 65$ billion can be attributed to direct health care costs [2]. CHF is characterized by an erratic and difficult-to-predict course. A high percentage of incurred costs are due to high readmission rates, as well as the high number of comorbidities [3].

Changes in physiological parameters such as weight, heart rate, blood pressure, and pulse oximetry may precede cardiac events. Signaling such changes through telemonitoring may enable physicians to intervene before the patient needs hospitalization or an emergency department (ED) visit [4]. Telemonitoring has been proposed as a possible strategy to tackle the challenges that CHF brings to health systems, most notably, spare use of the utilization of expensive resources. Telemonitoring has the potential to prevent hospital readmissions, thereby saving costs and improving the quality of life of these patients [5].

The technology and quality of care for patients with CHF have been evolving, which has resulted in a variety of telemonitoring programs consisting of different elements for different populations. In addition, studies on the effect of telemonitoring on health care utilization differ widely according to cointerventions, time horizons, and outcome measures. Such parameters may affect the effectiveness of telemonitoring and the comparability of studies [6]. This heterogeneity at many levels results in debates concerning the effectiveness of telemonitoring.

Studies on telemonitoring have shown mixed results with respect to health care utilization. Although some studies showed a decline, others showed no significant differences or even an increase in health care utilization. Many studies have included cointerventions within the telemonitoring intervention. Structured telephone support (STS) has often been incorporated [7-9]. As STS has been reported to reduce the number of heart failure-related hospitalizations [10], the effects found in studies that use these two interventions simultaneously may not be solely attributed to the telemonitoring program. A Cochrane study from 2015 [10] concluded that, although telemonitoring can reduce heart failure-related admissions, telemonitoring programs were not able to reduce the risk of all-cause hospitalization. A variety of systematic reviews have been performed, mostly exclusively including randomized controlled trials (RCTs). Bashi et al [11] performed an overview of systematic reviews and found that 11 of 19 systematic reviews only included RCTs. Although RCTs are considered as the gold standard for research purposes, observational studies also have merits that should not be ignored. For example, they provide a sense of the real world as opposed to experimental RCT settings [12]. Especially for diseases such as heart failure, which is known for a high degree of multimorbidity [13], RCTs may be limited by their strict inclusion and exclusion criteria. In addition, the vast majority of previous systematic reviews have only analyzed the effect of telemonitoring on hospitalizations and mortality $[10,11]$. Thus, limited information is available on the effect of telemonitoring programs on other outcomes, such as ED visits, length of stay, and other forms of health care utilization.

\section{Objective}

We therefore performed a systematic review on the effect of telemonitoring for $\mathrm{CHF}$ on health care utilization, including both RCTs and observational studies. By including a wide variety of telemonitoring programs and outcome measures, we aim to identify the various aspects and broad impact of telemonitoring programs on the health care utilization of patients with CHF.

\section{Methods}

\section{Search Strategy}

PubMed, Web of Science, and Embase (Ovid) databases were searched. All authors were consulted for additional eligible studies. A detailed description of our search strategy for each database can be found in Multimedia Appendix 1. We excluded articles published before January 1, 2010 because technology and insights have rapidly evolved over the past 10 years. Our search was updated until August 4, 2020 according to the method described by Bramer [14]. Removal of duplications was performed according to the method described by Bramer et al [15].

\section{Eligibility Criteria}

Telemonitoring includes a wide variety of definitions and descriptions. Generally, telemonitoring refers to the use of telecommunication to assist in the transmission of medical information and services between health care providers and patients [16]. This definition also includes STS. Many studies do not explicitly distinguish between invasive and noninvasive telemonitoring techniques. However, this review explicitly focuses on noninvasive techniques exclusively because there are significant differences between implanted monitoring devices and noninvasive telemonitoring in terms of costs and eligibility of patients [17]. Thus, we defined telemonitoring as the noninvasive application- or web-based collection and transfer of physiological data, aimed at improving quality of life or decreasing health care utilization in patients with heart failure, or both.

We included peer-reviewed studies that reported direct or indirect measurements of health care utilization such as hospitalizations, ED visits, length of stay, days of hospitalization, visits, and health care costs. We did not apply exclusion parameters concerning study design to include all available evidence. Therefore, we included RCTs, nonrandomized trials, and studies on observational data. However, we excluded studies that were not based on original data such as Markov models because these studies are based on assumptions rather than empirical data. Studies with regular telephone support initiated by nurses or health care providers (without medical indication) were excluded to focus on the 
effect of telemonitoring as opposed to the combined effect of telemonitoring and STS. We excluded studies from countries not belonging to the Organization for Economic Co-operation and Development to improve homogeneity in terms of the socioeconomic characteristics of the populations. Finally, studies that were not published in either English or Dutch were included. Textbox 1 presents the inclusion and exclusion criteria.

Textbox 1. Inclusion and exclusion criteria.

Inclusion criteria

- Telemonitoring equipment within program assessed physiological parameters.

- Telemonitoring measures were shared with at least one health care provider.

- $\quad$ Patients are living independently and are allowed to have access to home care services but should not be admitted to a nursing home during the intervention.

- Subjects have been diagnosed with chronic heart failure.

- Outcomes included direct or indirect measures of health care utilization.

- $\quad$ Paper was a peer-reviewed publication.

\section{Exclusion criteria}

- Study was performed in countries not belonging to the Organization for Economic Co-operation and Development.

- $\quad$ Publication date was before January 1, 2010.

- $\quad$ Telemonitoring program included structured telephone support.

- Intervention program used invasive telemonitoring (eg, CardioMEMS).

- Study was not available in the English or Dutch language.

- No quantitative data with accompanied statistical analysis or measure of statistical significance was reported.

- $\quad$ Health care utilization measure was not reported separately (eg, combined end point with death).

\section{Study Selection and Data Extraction}

Titles and abstracts were independently screened by 2 researchers (SLA and TEPR) for eligibility. In cases where no conclusive decision could be made, studies were included for full-text screening. Full-text screening was performed in duplicate and independently by SLA and TEPR. Disagreements were resolved by discussion until consensus was achieved or consultation with a coauthor (RBK or SAVD). Thereafter, data were extracted by one researcher (SLA) for both CHF-specific health care utilization and all-cause utilization because a reduction in CHF-specific health care utilization may not translate into an overall reduction [10]. For each study, the effect of telemonitoring on health care utilization was determined by extracting data on hospitalizations, ED visits, length of stay, days of hospitalization, visits, and health care costs. We determined the statistical significance of these results using a cut-off value of $P<.05$. If no $P$ value was reported, we deemed the effect statistically significant if the reported CI did not include 1.00 .

\section{Risk of Bias Assessment}

The risk of bias assessment was performed by SLA and TEPR. For the risk of bias assessment, we used the Cochrane risk-of-bias tool for randomized trials [18] and Risk of Bias in Non-randomized Studies of Interventions tools (Cochrane) [19] for RCTs and all remaining studies, respectively. The studies were assessed in duplicate and independently. Subsequently, the individual assessments were compared for possible discrepancies. When discrepancies were observed, the studies and corresponding assessments were discussed until a consensus was reached.

\section{Results}

\section{Overview}

Our search strategy identified 3770 unique studies, which were then screened in the title and abstract. This resulted in 99 remaining studies. After the full-text screening, 29 studies were included. Figure 1 shows the flow diagram. The main reason for exclusion after full-text screening was because STS was a part of the intervention $(19 / 70,27 \%)$, followed by a lack of measuring and transmitting physiological parameters in the program $(17 / 70,24 \%)$. Most studies $(11 / 29,38 \%)$ originated in the United States. 
Figure 1. PRISMA (Preferred Reported Items for Systematic Reviews and Meta-Analyses) flowchart. OECD: Organization for Economic Co-operation and Development.
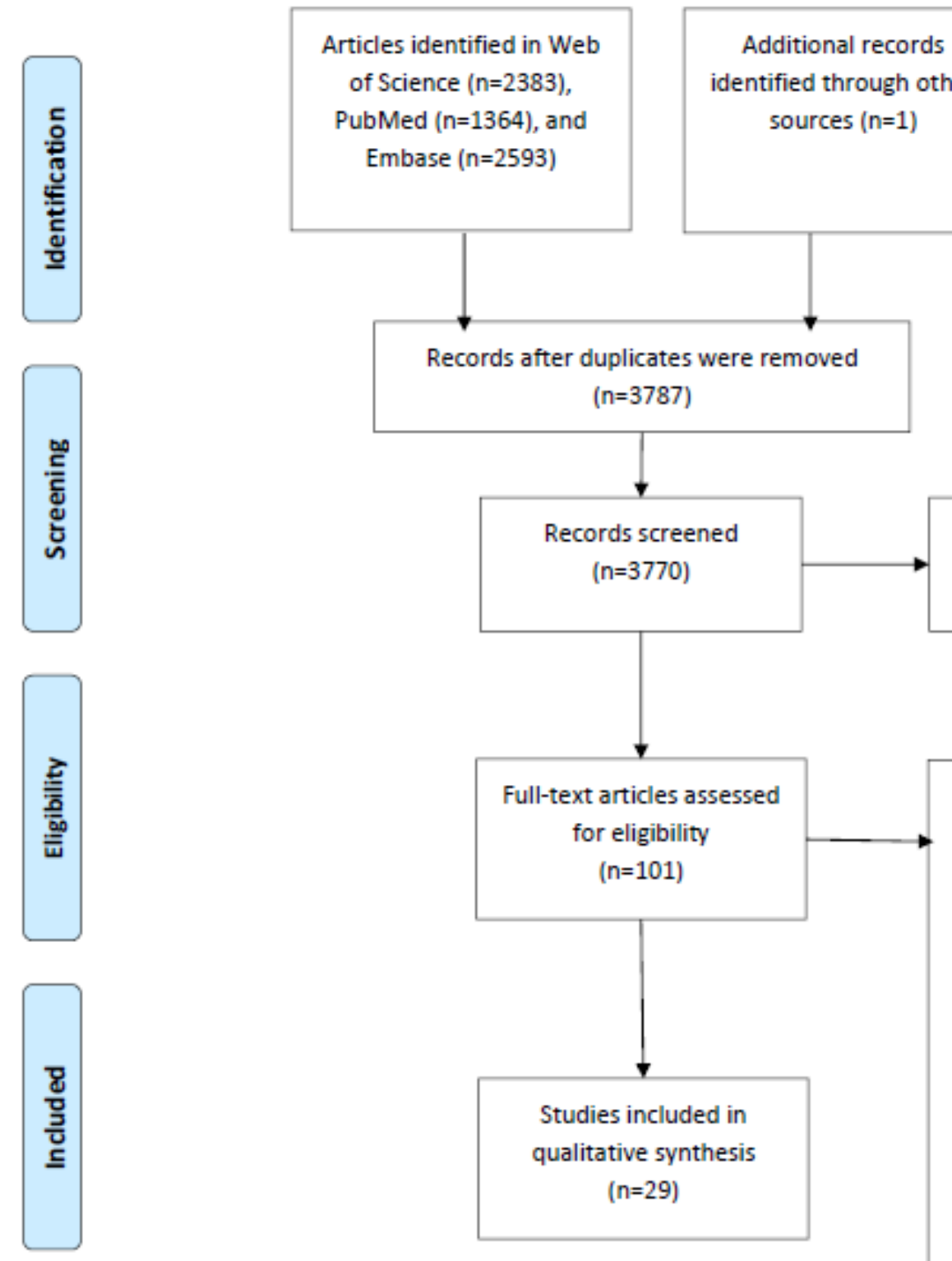

Articles identified in Web

of Science $(n=2383)$

Med $(n=1364)$, an

Records excluded

$(n=3686)$

Full-text articles excluded, due to

the following:

$(n=72)$

- No full text: 17

- Not published in the English or Dutch language: 1

- Did not measure and transmit any physiological parameters: 17

- Included structured telephone support in the intervention program: 21

- Study performed in countries not belonging to OECD: 2

- No original data (Markov model): 4

- Invasive telemonitoring: 2

- Did not report utilization: 1

- Did not assess telemonitoring effect: 1

- No statistical test performed: 6

\section{Characteristics of Included Studies}

Table 1 presents an overview of study and program characteristics. A detailed description of the studies and their characteristics are presented in Table 2. Telemonitoring programs showed a high degree of heterogeneity in terms of physiological variables measured as shown in Multimedia Appendix 2 [4,20-47]. All studies included weight as a parameter, whereas only 4 included electrocardiography measures as a physiological parameter [20-23]. The studies used 10 unique combinations of physiological parameters. In addition, 2 studies included other measures such as lung fluid [24] and body composition [25], which were acquired by noninvasive means. Most programs $(n=16)$ used telemonitoring in addition to usual care; regular check-up visits were still scheduled [4,22,24,26-38]. One study [20] stated that telemonitoring was used as a substitute for these visits, and 12 studies did not elaborate on this matter. The follow-up period of the studies ranged between 1 and 89 months, with most studies having a follow-up period of 6 months. Follow-up was consistent with the duration of the telemonitoring program itself, with the exception of 2 studies that also included a follow-up after the telemonitoring program was completed [24,39]. 
Table 1. Overview of the characteristics of included studies $(\mathrm{N}=29)$.

\begin{tabular}{|c|c|}
\hline Study characteristics & Studies, n (\%) \\
\hline \multicolumn{2}{|l|}{ Country } \\
\hline Belgium & $2(7)$ \\
\hline Canada & $2(7)$ \\
\hline Denmark & $1(3)$ \\
\hline Finland & $1(3)$ \\
\hline Israel & $2(7)$ \\
\hline Italy & $3(10)$ \\
\hline Japan & $1(3)$ \\
\hline Netherlands & $3(10)$ \\
\hline Spain & $1(3)$ \\
\hline Sweden & $1(3)$ \\
\hline United Kingdom & $1(3)$ \\
\hline United States & $11(38)$ \\
\hline \multicolumn{2}{|c|}{ Physiological parameter assessed } \\
\hline Weight & $29(100)$ \\
\hline Blood pressure & $23(79)$ \\
\hline Pulse oximetry & $7(24)$ \\
\hline Heart rate & $20(69)$ \\
\hline Symptom questions & $14(48)$ \\
\hline $\mathrm{ECG}^{\mathrm{a}}$ & $4(14)$ \\
\hline \multicolumn{2}{|c|}{ Number of patients enrolled in telemonitoring program } \\
\hline$<50$ & $8(28)$ \\
\hline $50-100$ & $9(31)$ \\
\hline $100-200$ & $9(31)$ \\
\hline$>200$ & $3(10)$ \\
\hline \multicolumn{2}{|c|}{ Follow-up period (months) } \\
\hline 1 & $1(3)$ \\
\hline 2 & $1(3)$ \\
\hline 3 & $4(14)$ \\
\hline 6 & $10(34)$ \\
\hline 9 & $1(3)$ \\
\hline 12 & $8(28)$ \\
\hline$>12$ & $4(14)$ \\
\hline
\end{tabular}

${ }^{a}$ ECG: electrocardiography. 
Table 2. Detailed characteristics of the included studies $(\mathrm{N}=29)$.

\begin{tabular}{|c|c|c|c|c|c|c|c|}
\hline \multirow[t]{2}{*}{ Study } & \multicolumn{7}{|c|}{ Study characteristics } \\
\hline & Country & Study design & Patient inclusion criteria & $\begin{array}{l}\mathrm{NYHA}^{\mathrm{a}} \\
\text { class }\end{array}$ & $\begin{array}{l}\text { Follow- } \\
\text { up period } \\
\text { (months) }\end{array}$ & $\begin{array}{l}\text { Intervention } \\
\text { group }(\mathrm{n})\end{array}$ & $\begin{array}{l}\text { Control } \\
\text { group, } \mathrm{n}\end{array}$ \\
\hline Amir [24] & United States & Prospective & $\begin{array}{l}\text { Stage } \mathrm{C} \mathrm{CHF}^{\mathrm{b}} \text { with hospital } \\
\text { admission for acute } \mathrm{HF}^{\mathrm{c}}\end{array}$ & $\underline{\mathrm{d}}^{\mathrm{d}}$ & 6 & 50 & $\mathrm{~N} / \mathrm{A}^{\mathrm{e}}$ \\
\hline Bakhshi [42] & Israel & $\begin{array}{l}\text { Comparison of group with } \\
\text { telemonitoring versus group } \\
\text { without telemonitoring }\end{array}$ & $\begin{array}{l}\text { Primary diagnosis CHF index } \\
\text { hospitalization or patients seen } \\
\text { by emergency room, internal } \\
\text { medicine, and cardiology spe- } \\
\text { cialists }\end{array}$ & - & 6 & 44 & 16 \\
\hline Delaney [43] & United States & $\mathrm{RCT}^{\mathrm{f}}$ & $\begin{array}{l}\text { Recently discharged from home } \\
\text { care, eligible, and previously } \\
\text { hospitalized for HF }\end{array}$ & III: $95(95 \%)$ & 3 & 50 & 50 \\
\hline Dendale [44] & Belgium & RCT & $\begin{array}{l}\text { Hospitalized for fluid overload } \\
\text { due to } \mathrm{HF}\end{array}$ & $\begin{array}{l}\text { Mean NY- } \\
\text { HA class: } \\
3.0\end{array}$ & 6 & 80 & 80 \\
\hline $\begin{array}{l}\text { Domingo } \\
{[26]}\end{array}$ & Spain & $\mathrm{RCT}$ & $\begin{array}{l}\text { Outpatients attended in the HF } \\
\text { unit with NYHA classes II-IV }\end{array}$ & $\begin{array}{l}\text { II: } 75(82 \%) \\
\text { and III: } 17 \\
(18 \%)\end{array}$ & 12 & 44 & 48 \\
\hline $\begin{array}{l}\text { Eilat- } \\
\text { Tsanani [45] }\end{array}$ & Israel & Before-after & $\begin{array}{l}\text { NYHA classes II-IV with at } \\
\text { least } 3 \text { admissions within a } 6 \text { - } \\
\text { month period }\end{array}$ & $\begin{array}{l}\text { II: } 74 \\
(52.5 \%) ; \text { III: } \\
55(39 \%) ; \\
\text { and IV: } 12 \\
(8.5 \%)\end{array}$ & 12 & 141 & N/A \\
\hline Frederix [39] & Belgium & RCT & $\begin{array}{l}\text { Patients participated in the } \\
\text { TEMA-HF }{ }^{\mathrm{g}} \text { study by Dendale } \\
\text { et al [44] }\end{array}$ & $\begin{array}{l}\text { Mean NY- } \\
\text { HA class: } \\
3.0\end{array}$ & 89 & 80 & 80 \\
\hline Hoban [27] & United States & RCT & $\begin{array}{l}\text { HF diagnosis care from home } \\
\text { health care agency }\end{array}$ & - & 3 & 40 & 40 \\
\hline $\begin{array}{l}\text { Kotooka } \\
{[25]}\end{array}$ & Japan & RCT & $\begin{array}{l}\text { Discharge following admission } \\
\text { for acute HF or decompensated } \\
\text { chronic HF }\end{array}$ & $\begin{array}{l}\text { II: } 142 \\
(78.5 \%) ; \text { III: } \\
39(21.5 \%)\end{array}$ & 31 & 90 & 91 \\
\hline $\begin{array}{l}\text { Koulaouzidis } \\
\text { [28] }\end{array}$ & United Kingdom & $\begin{array}{l}\text { Retrospective comparison } \\
\text { with patients that declined }\end{array}$ & $\begin{array}{l}\text { Newly diagnosed (outpatient) } \\
\text { patients with HF }\end{array}$ & $\begin{array}{l}\text { II: } 238 \\
(52.5 \%) \text { and } \\
\text { III: } 215 \\
(47.5 \%)\end{array}$ & 12 & 124 & 329 \\
\hline Kraai [20] & Netherlands & Multicenter RCT & $\begin{array}{l}\text { Admitted to intensive care or } \\
\text { cardiology ward or visited out- } \\
\text { patient HF- clinic and in need } \\
\text { of treatment }\end{array}$ & $\begin{array}{l}\text { II:39 }(22 \%) \\
\text { III:100 } \\
(58.5 \%) \text { and } \\
\text { IV:33 } \\
(18.6 \%)\end{array}$ & 9 & 94 & 83 \\
\hline Lyngå [29] & Sweden & $\mathrm{RCT}$ & $\begin{array}{l}\text { Hospitalized with NYHA } \\
\text { classes III-IV }\end{array}$ & $\begin{array}{l}\text { III: } 309 \\
(96.9 \%) ; \text { IV: } \\
10(3.1 \%)\end{array}$ & 12 & 166 & 153 \\
\hline Maeng [30] & United States & $\begin{array}{l}\text { Retrospective within pa- } \\
\text { tients }\end{array}$ & Have a diagnosis of $\mathrm{CHF}$ & - & 70 & 541 & N/A \\
\hline Olivari [21] & Italy & $\mathrm{RCT}$ & $\begin{array}{l}\text { Discharge from hospital after } \\
\text { acute HF within the past } 3 \\
\text { months }\end{array}$ & $\begin{array}{l}\text { II: } 163 \\
(48.1 \%) ; \text { III: } \\
\text { 159 }(46.9 \%) \\
\text { IV: } 17(5 \%)\end{array}$ & 12 & 229 & 119 \\
\hline Park [40] & United States & $\begin{array}{l}\text { Prospective comparison be- } \\
\text { tween hospital and national } \\
\text { readmission rates }\end{array}$ & $\begin{array}{l}\text { Admitted for a diagnosis of } \\
\text { acute HF }\end{array}$ & - & 1 & 58 & N/A \\
\hline
\end{tabular}




\begin{tabular}{|c|c|c|c|c|c|c|c|}
\hline \multirow[t]{2}{*}{ Study } & \multicolumn{7}{|c|}{ Study characteristics } \\
\hline & Country & Study design & Patient inclusion criteria & $\begin{array}{l}\text { NYHA }^{\mathrm{a}} \\
\text { class }\end{array}$ & $\begin{array}{l}\text { Follow- } \\
\text { up period } \\
\text { (months) }\end{array}$ & $\begin{array}{l}\text { Intervention } \\
\text { group }(\mathrm{n})\end{array}$ & $\begin{array}{l}\text { Control } \\
\text { group, } \mathrm{n}\end{array}$ \\
\hline Pedone [31] & Italy & $\mathrm{RCT}$ & $\begin{array}{l}\text { Diagnosis of } \mathrm{HF} \text { and aged }>64 \\
\text { years }\end{array}$ & $\begin{array}{l}\text { II: } 29(32 \%) \text {; } \\
\text { III: } 51 \\
\text { (57\%); IV: } \\
10(11 \%)\end{array}$ & 6 & 50 & 46 \\
\hline Riley [41] & United States & $\begin{array}{l}\text { Prospective comparison with } \\
\text { matched controls }\end{array}$ & $\begin{array}{l}\text { HF admission diagnosis in the } \\
\text { electronic health record }\end{array}$ & - & 6 & 45 & 45 \\
\hline Seto [22] & Canada & RCT & $\begin{array}{l}\text { Ambulatory patients diagnosed } \\
\text { with HF }\end{array}$ & $\begin{array}{l}\text { II: } 43 \text { (43\%); } \\
\text { II-III: } 11 \\
\text { (11\%); III: } \\
42(42 \%) ; \\
\text { IV: } 4(4 \%)\end{array}$ & 6 & 50 & 50 \\
\hline Soran [32] & United States & $\mathrm{RCT}$ & $\begin{array}{l}\text { Diagnosis of HF in Medicare } \\
\text { data and hospitalized for HF } \\
\text { within } 6 \text { months }\end{array}$ & $\begin{array}{l}\text { II: } 183 \\
\text { (58.1\%); III: } \\
132(41.9 \%)\end{array}$ & 6 & 160 & 155 \\
\hline $\begin{array}{l}\text { Tompkins } \\
\text { [33] }\end{array}$ & United States & $\mathrm{RCT}$ & Diagnosis of $\mathrm{HF}$ & - & 6 & 193 & 197 \\
\hline $\begin{array}{l}\text { Van der } \\
\text { Burg [34] }\end{array}$ & Netherlands & $\begin{array}{l}\text { Retrospective before-after } \\
\text { study }\end{array}$ & NYHA classes III-IV & - & 36 & 177 & N/A \\
\hline $\begin{array}{l}\text { Veenstra } \\
{[35]}\end{array}$ & Netherlands & $\begin{array}{l}\text { Prospective prestudy and } \\
\text { poststudy without controls }\end{array}$ & $\begin{array}{l}\text { Discharged after admission for } \\
\text { HF or outpatient visit and NY- } \\
\text { HA classes II-IV }\end{array}$ & $\begin{array}{l}\text { II: } 28 \\
\text { (27.5\%); III: } \\
57(55.9 \%) \text {; } \\
\text { IV: } 17 \\
(16.7 \%)\end{array}$ & 12 & 102 & N/A \\
\hline $\begin{array}{l}\text { Vestergaard } \\
{[46]}\end{array}$ & Denmark & RCT with economic analysis & $\begin{array}{l}\text { Diagnosed with HF according } \\
\text { to national guidelines and NY- } \\
\text { HA classes II-IV }\end{array}$ & $\begin{array}{l}\text { Missing:13 } \\
\text { (4.7\%); II: } \\
147(53.6 \%) \text {; } \\
\text { III: 92 } \\
(33.6 \%) \text {; IV: } \\
22(8 \%)\end{array}$ & 12 & 134 & 140 \\
\hline Villani [23] & Italy & RCT & $\begin{array}{l}\text { NYHA classes III or IV during } \\
\text { hospital stay and high risk of } \\
\text { early rehospitalization at dis- } \\
\text { charge }\end{array}$ & $\begin{array}{l}\text { Mean NY- } \\
\text { HA: } 3.01\end{array}$ & 12 & 40 & 40 \\
\hline $\begin{array}{l}\text { Vuorinen } \\
\text { [47] }\end{array}$ & Finland & RCT & $\begin{array}{l}\text { Left ventricular ejection frac- } \\
\text { tion }<35 \%, N Y H A \geq 2 \text {, and } \\
\text { needed regular follow-up }\end{array}$ & $\begin{array}{l}\text { II: } 36(38 \%) \text {; } \\
\text { III: } 55 \\
(59 \%) ; \text { IV: } 3 \\
(3 \%)\end{array}$ & 6 & 47 & 47 \\
\hline Ware [36] & Canada & Before-after study & $\begin{array}{l}\text { Diagnosed with HF and visited } \\
\text { the HF clinic }\end{array}$ & $\begin{array}{l}\text { SII: } 143 \\
(45.4 \%)\end{array}$ & 6 & 315 & N/A \\
\hline $\begin{array}{l}\text { White- } \\
\text { Williams [4] }\end{array}$ & United States & $\begin{array}{l}\text { Retrospective study with } \\
\text { controls }\end{array}$ & $\begin{array}{l}\text { Discharged from hospital stay } \\
\text { and admitted to a home health } \\
\text { care agency }\end{array}$ & - & 3 & 29 & 17 \\
\hline $\begin{array}{l}\text { Williams } \\
\text { [37] }\end{array}$ & United States & $\begin{array}{l}\text { Retrospective study with } \\
\text { matched controls }\end{array}$ & $\begin{array}{l}\text { Medicare eligible, had a diagno- } \\
\text { sis of HF, and experienced a } \\
\text { recent ( } 2 \text { days) discharge from } \\
\text { hospital }\end{array}$ & - & 2 & 105 & 105 \\
\hline
\end{tabular}




\begin{tabular}{|c|c|c|c|c|c|c|c|}
\hline \multirow[t]{2}{*}{ Study } & \multicolumn{7}{|c|}{ Study characteristics } \\
\hline & Country & Study design & Patient inclusion criteria & $\begin{array}{l}\text { NYHA }^{\mathrm{a}} \\
\text { class }\end{array}$ & $\begin{array}{l}\text { Follow- } \\
\text { up period } \\
\text { (months) }\end{array}$ & $\begin{array}{l}\text { Intervention } \\
\text { group (n) }\end{array}$ & $\begin{array}{l}\text { Control } \\
\text { group, } \mathrm{n}\end{array}$ \\
\hline Zan [38] & United States & $\begin{array}{l}\text { Prospective study with } \\
\text { matched controls }\end{array}$ & $\begin{array}{l}\text { Participants recruited from the } \\
\text { outpatient clinic. Patients admit- } \\
\text { ted to hospital program were } \\
\text { excluded }\end{array}$ & $\begin{array}{l}\text { Intervention } \\
\text { group: I: } 5 \\
\text { (24\%); II: } 9 \\
(43 \%) \text {; III: } 7 \\
(33 \%)\end{array}$ & 3 & 21 & 20 \\
\hline
\end{tabular}

${ }^{\mathrm{a}}$ NYHA: New York Heart Association.

${ }^{\mathrm{b}} \mathrm{CHF}$ : chronic heart failure.

${ }^{\mathrm{c}} \mathrm{HF}$ : heart failure.

${ }^{\mathrm{d}}$ Data not reported.

${ }^{\mathrm{e}} \mathrm{N} / \mathrm{A}$ : not applicable.

${ }^{\mathrm{f}} \mathrm{RCT}$ : randomized controlled trial.

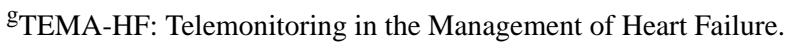

Patient inclusion criteria for previous hospitalizations differed among the studies. If a hospital admission was required for inclusion, this was most often $(\mathrm{n}=10)$ within 7 days of discharge [4,23,24,29,35,37,39-42]. Inclusion was started within 1 month of index hospitalization for 2 studies [20,25], and 5 studies [21,32,43-45] included patients within 6 months. Most studies $(n=12) \quad[22,26-28,30,31,33,34,36,38,46,47]$ did not specify requirements regarding previous admissions for $\mathrm{CHF}$ or included patients from outpatient clinics. New York Heart Association (NYHA) Class II was most often reported $(n=9)$ as the NYHA Classification with most patients $[21,22,25,26,28,32,38,45,46]$. NYHA Class III was reported in 8 studies as the dominant class [20,23,29,31,35,43,44,47].

\section{Risk of Bias Assessment}

We found that most RCTs $(12 / 16,75 \%)$ showed at least some bias concerns, with 2 RCTs having a high risk of bias [21,27].

The main reasons for some concerns in the RCTs were related to the randomization process. In addition, $75 \%$ (9/12) of nonrandomized studies were assessed as having a serious or critical risk of bias. No nonrandomized studies were assessed as having a low risk of bias, which indicates that the strength of evidence from none of the included nonrandomized studies is equivalent to a well-performed RCT [19]. Bias due to confounding was the domain that most often resulted in studies being assessed with a serious or critical risk for bias. Table 3 shows the consolidated overall bias assessments. A detailed overview of the risk of bias assessment of all studies can be found in Multimedia Appendix 3 [20-23,25-27,29,31-33,39,43,44,46,47] and Multimedia Appendix 4 [4,24,28,30,34-38,40-42,45]. 
Table 3. Summary of risk of bias assessment for randomized controlled trials and nonrandomized studies.

\begin{tabular}{|c|c|}
\hline Study & Result of bias risk assessment \\
\hline \multicolumn{2}{|l|}{ Randomized controlled trials ${ }^{\mathrm{a}}$} \\
\hline Delaney (2013) [43] & Some concerns \\
\hline Dendale (2014) [44] & Some concerns \\
\hline Domingo (2011) [26] & Some concerns \\
\hline Hoban (2013) [27] & High risk \\
\hline Kotooka (2018) [25] & Low risk \\
\hline Kraai (2016) [20] & Low risk \\
\hline Lyngå (2012) [29] & Some concerns \\
\hline Frederix (2018) [39] & Low risk \\
\hline Olivari (2018) [21] & High risk \\
\hline Pedone (2015) [31] & Some concerns \\
\hline Seto (2012) [22] & Some concerns \\
\hline Soran (2010) [32] & Some concerns \\
\hline Tompkins (2012) [33] & Some concerns \\
\hline Vestergaard (2020) [46] & Low risk \\
\hline Villani (2014) [23] & Some concerns \\
\hline Vuorinen (2014) [47] & Some concerns \\
\hline \multicolumn{2}{|l|}{ Nonrandomized studies ${ }^{b}$} \\
\hline Amir (2017) [24] & Moderate risk \\
\hline Bakhshi (2011) [42] & Serious risk \\
\hline Eilat-Tsanani (2015) [45] & Critical risk \\
\hline Koulaouzidis (2019) [28] & Serious risk \\
\hline Maeng (2014) [30] & Critical risk \\
\hline Park (2019) [40] & Critical risk \\
\hline Riley (2015) [41] & Moderate risk \\
\hline Van der Burg (2020) [34] & Serious risk \\
\hline Veenstra (2015) [35] & Critical risk \\
\hline Ware (2020) [36] & Serious risk \\
\hline White-Williams (2015) [4] & Serious risk \\
\hline Williams (2016) [37] & Moderate risk \\
\hline Zan (2015) [38] & Serious risk \\
\hline
\end{tabular}

${ }^{\mathrm{a}}$ Assessed using Cochrane risk-of-bias tool for randomized trials.

${ }^{\mathrm{b}}$ Assessed using Risk of Bias in Non-randomized Studies of Interventions tool.

Effects of Telemonitoring on Health Care Utilization

\section{Overview}

Telemonitoring studies used many different outcome measures, the most common of which were hospitalizations, ED visits, and health care costs. Whereas some studies report the effects of telemonitoring on specific CHF outcomes, for example, heart failure readmissions, other studies only report the effect on all-cause health care utilization. The type of analysis and effect measures differed widely among studies, with hazard ratios, odds ratios, 2-tailed $t$ tests, and incidence rates being reported. Table 4 shows the (statistically significant) effects of telemonitoring on health care utilization. 
Table 4. Effect of telemonitoring programs on health care utilization.

\begin{tabular}{|c|c|c|c|c|c|c|c|c|c|c|c|c|}
\hline \multirow[t]{3}{*}{ Study } & \multicolumn{12}{|c|}{ Outcome measure } \\
\hline & \multicolumn{2}{|c|}{ Hospitalization } & \multicolumn{2}{|c|}{$\begin{array}{l}\text { Number of emergency } \\
\text { department visits }\end{array}$} & \multicolumn{2}{|c|}{$\begin{array}{l}\text { Length of } \\
\text { stay }\end{array}$} & \multicolumn{2}{|c|}{ Days of hospitalization } & \multicolumn{2}{|c|}{ Visits $^{\mathrm{a}}$} & \multicolumn{2}{|c|}{$\begin{array}{l}\text { Health care } \\
\text { costs }\end{array}$} \\
\hline & $\mathrm{HF}^{\mathrm{b}}$ & $\mathrm{AC}^{\mathrm{c}}$ & $\mathrm{HF}$ & $\mathrm{AC}$ & $\mathrm{HF}$ & $\mathrm{AC}$ & $\mathrm{HF}$ & $\mathrm{AC}$ & $\mathrm{HF}$ & $\mathrm{AC}$ & $\mathrm{HF}$ & $\mathrm{AC}$ \\
\hline $\operatorname{Amir}(2017)$ [24] & $-^{\mathrm{d}}$ & $+^{\mathrm{e}}$ & - & - & - & - & - & - & - & - & - & - \\
\hline Bakhshi (2011) [42] & - & - & - & - & - & $={ }^{\mathrm{f}}$ & - & - & - & - & - & - \\
\hline Delaney (2013) [43] & $=$ & + & - & - & - & - & - & - & - & - & - & - \\
\hline Dendale (2014) [44] & $=$ & $=$ & - & - & - & - & - & $=$ & - & - & - & $=$ \\
\hline Domingo $(2011)^{\mathrm{g}}[26]$ & $=$ & - & - & - & - & - & $=$ & - & $x^{h}$ & - & - & - \\
\hline Eilat-Tsanani (2015) [45] & $=$ & + & - & $=$ & - & - & - & + & - & $=$ & - & - \\
\hline Frederix (2018) [39] & - & - & - & - & - & - & + & $=$ & - & - & $=$ & $=$ \\
\hline Hoban (2013) [27] & - & $=$ & - & - & - & - & - & - & - & - & - & - \\
\hline Kotooka (2018) [25] & $=$ & $=$ & - & - & - & - & - & - & - & - & - & - \\
\hline Koulaouzidis (2019) [28] & $=$ & $=$ & - & - & - & - & + & $=$ & - & - & - & - \\
\hline Kraai (2016) [20] & $=$ & $=$ & - & - & - & - & - & - & + & $=$ & - & $=$ \\
\hline Lyngå (2012) [29] & $=$ & $=$ & - & - & - & - & $=$ & - & - & - & - & - \\
\hline Maeng (2014) [30] & - & + & - & - & - & - & - & - & - & - & - & + \\
\hline Olivari $(2018)^{\mathrm{i}}[21]$ & $=$ & $=$ & - & $=$ & $=$ & $=$ & - & - & - & - & - & - \\
\hline Park (2019) [40] & - & $=$ & - & - & - & - & - & - & - & - & - & - \\
\hline Pedone (2015) [31] & $=$ & + & - & - & - & - & - & - & - & - & - & - \\
\hline Riley $(2015)^{\mathrm{j}}[41]$ & - & $=$ & - & - & - & - & - & $=$ & - & - & - & - \\
\hline Seto (2012) [22] & - & $=$ & - & $=$ & - & - & - & $=$ & $x$ & - & - & - \\
\hline Soran (2010) [32] & - & - & - & - & - & - & - & - & - & - & - & $x$ \\
\hline Tompkins (2012) [33] & - & $=$ & - & $=$ & - & $=$ & - & $=$ & - & $x$ & - & $=$ \\
\hline Van der Burg (2020) [34] & - & + & - & - & - & - & - & + & - & - & - & + \\
\hline Veenstra (2015) [35] & + & - & - & - & - & - & - & - & - & - & - & - \\
\hline Vestergaard (2020) [46] & - & - & - & - & - & - & - & - & - & - & - & + \\
\hline Villani (2014) [23] & + & - & - & + & - & - & - & - & - & $x$ & - & $x$ \\
\hline Vuorinen (2014) [47] & - & - & - & - & - & - & $=$ & - & $x$ & $x$ & - & - \\
\hline Ware (2020) [36] & + & + & $=$ & $=$ & $=$ & $=$ & - & - & $=$ & - & - & - \\
\hline White-Williams (2015) [4] & - & $=$ & - & $=$ & - & - & - & - & - & - & - & - \\
\hline Williams (2016) [37] & - & $=$ & - & - & - & - & - & - & - & $x$ & - & $x$ \\
\hline Zan (2015) [38] & - & $=$ & - & $=$ & - & - & - & - & - & - & - & - \\
\hline
\end{tabular}

${ }^{\mathrm{a}}$ Visits include all nonemergency department outpatient visits or consultations with health care provider contacts.

${ }^{\mathrm{b}} \mathrm{HF}$ : heart failure-related.

${ }^{\mathrm{c}} \mathrm{AC}$ : all-cause.

${ }^{\mathrm{d}}$ Data not reported.

${ }^{\mathrm{e}}$ Indicates statistically significant $(P<.05)$ reduction in outcome measure.

${ }^{\mathrm{f}}$ Indicates not statistically significant $(P>.05)$ on outcome measure.

${ }^{\mathrm{g}}$ Analysis based on the comparison between Motiva and Motiva plus.

${ }^{\mathrm{h}}$ Indicates statistically significant $(P<.05)$ increase in outcome measure.

${ }^{\mathrm{i}}$ Analysis based on intention-to-treat analysis.

${ }^{\mathrm{j}}$ Analysis based on matched-cohort analysis. 


\section{Hospitalization}

Hospitalization was the most commonly reported outcome $(24 / 29,83 \%$ of studies). Out of 24 studies, 9 (38\%) showed a statistically significant reduction in hospitalizations associated with telemonitoring. The remaining studies $(15 / 24,63 \%)$ found no effect on hospitalization. When excluding all studies with a high, critical, or serious risk of bias $(n=11), 31 \%(4 / 13)$ of studies reported a statistically significant reduction in hospitalizations [23,24,31,43]. Although the number of studies reporting a statistically significant effect was limited, the effects found in these studies were clinically relevant. Amir et al [24] reported a temporary reduction of hospitalizations of $87 \%$ during the intervention. However, hospitalization increased by $79 \%$ when the intervention ended. Delaney et al [43] found that hospitalization rates were $19 \%$ and $38 \%$ in the intervention and control groups, respectively. Pedone et al [31] reported an incidence rate of 0.30 (95\% CI 0.12-0.67), favoring the intervention group. Villani et al [23] found that the control group had almost twice the number of hospitalizations compared with the intervention group. We did not find a parameter in the study characteristics that consistently differed from the other studies that showed no effect of telemonitoring.

\section{ED Visits}

In total, 8 studies reported results related to the number of ED visits $[4,21-23,33,36,38,45] ; 7(88 \%)$ reported no statistically significant effects of telemonitoring and $1(13 \%)$ found a significant effect, reporting $17 \mathrm{ED}$ visits in the control group $(n=40)$ compared with 6 ED visits in the intervention group $(\mathrm{n}=40)$ [23].

\section{Length of Stay}

The outcome measure length of stay was reported in 4 studies $[21,33,36,42]$. None of the included studies found a statistically significant effect of telemonitoring on the length of stay.

\section{Days of Hospitalization}

Days of hospitalization were reported in 10 studies, of which 8 $(80 \%)$ reported on all-cause days of hospitalization and $5(50 \%)$ on days of hospitalization specifically for heart failure. There were 2 studies that showed a reduction in all-cause days of hospitalization [34,45]. The 2 studies showing a decrease in heart failure-related days of hospitalization did not result in a statistically significant decrease in all-cause days of hospitalization [28,39].

\section{Visits}

A total of 9 studies reported outcome measures related to non-ED health care visits. These visits comprised outpatient visits [20,22,23,26,36,45,47], primary care visits [33,45], home visits by nurse [37,45], urgent care visits [33], and telephone contacts [47]. Most $(6 / 9,67 \%)$ of these studies found that their telemonitoring intervention was associated with a significant increase in non-ED health care visits [22,23,26,33,37,47]. A total of 2 studies found no difference [36,45], and 1 study [20] found a decrease in non-ED visits for heart failure specifically. However, this reduction of heart failure rates did not result in a statistically significant decrease in the number of visits. Two studies were considered as having a critical [45] or serious risk of bias [36]. Both of these studies reported no effect of telemonitoring on non-ED visits.

\section{Health Care Costs}

A comparison of health care costs between the intervention and control groups was performed in 10 studies. Out of these 10, 3 $(30 \%)$ studies showed a decrease in costs [30,34,46], $4(40 \%)$ studies showed no statistically significant difference $[20,33,39,44]$, and $3(30 \%)$ studies showed a negative impact of telemonitoring on health care costs [23,32,37]. Two studies, which we assessed as having a serious or critical risk of bias, reported statistically significant decreases in health care costs $[30,34]$. When a decrease in costs was realized by the telemonitoring program, this was mainly due to a reduction in hospitalizations or rehospitalizations [30,34]. In addition to a reduction in hospitalizations, Vestergaard et al [46] found a reduction in outpatient care costs in the telemonitoring group. Furthermore, 3 studies finding a reduction of health care costs reported $11 \%$ [30], 35\% [46], and 90\% [34] reductions in health care costs. When telemonitoring was associated with an increase in costs, this was due to an increase in outpatient visits [37], an overall increase in health care utilization [32], or the costs of the telemonitoring program itself [23].

Six studies included and explicitly mentioned the costs of (the development of) the intervention in their analyses $[20,23,30,32,37,46]$. These studies reported large differences in the costs of the intervention, mostly due to cointerventions and specific assumptions regarding program and development costs. Multimedia Appendix 5 [20,23,30,32,37,46] provides a description of the costs. In the study by Villani et al [23], a reduction in hospitalization costs could not offset the additional costs of the intervention. This indicates a substantial influence of program costs in determining the cost-effectiveness of telemonitoring programs. An important factor for cost differences was the high cost of developing a telemonitoring program for a selected population.

\section{Discussion}

\section{Principal Findings}

The goal of this review was to identify the broad impact and various aspects of telemonitoring programs for patients with $\mathrm{CHF}$ and their effects on health care utilization. Most studies showed no statistically significant effects of telemonitoring on overall hospitalizations (14/21, 67\%), ED visits (7/8, 88\%), length of stay $(4 / 4,100 \%)$, and days of hospitalization $(6 / 8$, $75 \%$ ). However, the remaining studies showed reductions in health care utilization for these measures. Overall, non-ED outpatient visits and health care costs were increased in $67 \%$ (6/9) of studies and $30 \%$ (3/10) of studies, respectively. The most ambiguously reported health care utilization measure was health care costs, which showed increases in 30\% (3/10) of the studies, decreases in $30 \%(3 / 10)$ of the studies, and no differences in $40 \%$ (4/10) of the studies. Heart failure-specific health care utilization measures showed similar results as the overall health care utilization measures.

We found a high degree of clinical diversity among the interventions, in terms of physiological parameters as well as 
the targeted populations. Although most telemonitoring programs were targeted at patients in NYHA Classes II-III, a variety of additional inclusion criteria were used. In addition to clinical diversity, methodological heterogeneity was also high, as can be observed by the varying risk of bias assessments.

Some of the studies included in this review suggested that telemonitoring may result in lower hospital admission rates, but most studies did not report such reductions. This mixed effect is consistent with the current literature [10,48]. In addition, we found no clear associations between the extracted study characteristics and the effect of telemonitoring and a reduction in hospitalizations. However, we found that $67 \%$ (6/9) of studies reported an increase in non-ED outpatient visits when telemonitoring was used.

Thus far, the effect of telemonitoring on non-ED visits has received little attention. However, the early warning designs of telemonitoring might also attract additional use of frontline services and false-positive alarms. An overview of systematic reviews [11] showed that 2 of 19 systematic reviews included non-ED visits in their outcomes [49,50]. These systematic reviews included 5 studies, 1 [51] of which can be classified as telemonitoring based on our definition. This RCT showed a substantial increase in non-ED visits and phone calls for telemonitoring compared with usual care. Our results suggest that this may be the case for most telemonitoring programs, as we found that $67 \%(6 / 9)$ of studies showed an increase in non-ED visits. One study found a decrease in heart failure-related non-ED visits. This study [20] explicitly stated that "patients allocated to the intervention group were only allowed to visit the cardiologist or HF-nurse in case of an absolute need for intervention." In addition, 83\% (5/6) of studies, which found an increase in visits, had telemonitoring as an additional component in addition to usual care [22,26,37,47], and 17\% (1/6) did not state this explicitly [33]. This stresses the need to treat telemonitoring as a substitute for regular care, rather than an addition to regular care, that is, if a reduction in health care utilization is the primary aim. Five studies reported an increase in non-ED visits and hospitalizations as outcome measures. Villani et al [23] found an increase in non-ED visits simultaneously with a reduction in hospitalizations. The other 4 studies [22,26,33,37] reported an increase in non-ED visits and found no effect on hospitalization. This indicates that additional visits can be a side effect of telemonitoring rather than a prerequisite for effective telemonitoring programs in terms of reduced health care utilization. Therefore, telemonitoring programs may become more cost-effective if they pay more attention to decreasing these visits.

The effect of telemonitoring on health care costs has been inconsistent across studies. Health care costs were severely affected by the costs of telemonitoring programs, which showed large differences. These differences were attributable to both assumptions regarding the development costs per patient and the actual cost differences. These cost differences can have a detrimental effect on the cost-effectiveness and financial viability of the program. No studies included indirect cost savings or expenses from a patient perspective, such as a reduction or increase in travel costs.

\section{Strength and Limitations}

A major strength of our study is the inclusion of various study designs and outcome measures for health care utilization. Therefore, this review offers a broader scope than other reviews [11]. The inherent weakness of including nonrandomized studies was the introduction of biases because of case mixes. This was demonstrated by the results of the Risk of Bias in Non-randomized Studies of Interventions tool, which showed a high degree of bias in the domain of baseline confounding. The exclusion of studies also using STS within their telemedicine program resulted in the exclusion of a significant number of studies. However, the exclusion of these studies also increases the relevance of our study for those interested in the stand-alone effect of telemonitoring on health care utilization.

The main limitation of this review is the lack of a meta-analysis and thus a limited ability to draw strong conclusions regarding the effect of telemonitoring on health care utilization. However, this review clearly shows why meta-analyses are difficult to perform on this subject and, if performed, should be interpreted with caution. We observed high heterogeneity within the study populations, telemonitoring programs, and outcomes. Therefore, a meta-analysis was not appropriate for this study. In addition, the results of meta-analyses of other studies may only be applicable to small subsets of populations, interventions, and outcomes and thus may not represent the true effect of telemonitoring on health care utilization because of limited external validity.

\section{Implications for Practice}

This study has several practical implications. The finding that telemonitoring often increases non-ED visits has consequences for the workload of outpatient clinics. These additional non-ED visits may occur because of a variety of reasons such as false positives, true positives, equipment malfunction, and whether telemonitoring is used as additional or substitute care. Detecting true positives is the primary aim, as it prevents more expensive health care utilization and improves the quality of life. Although false-positive alerts and equipment malfunctions may be reduced by improving technology and algorithms [52], our results suggest that addition instead of substitution is likely to remain, resulting in additional non-ED visits. Health care providers must be aware of this increase and adopt organizational structure of the outpatient clinic or find other ways to mitigate this increase, such as using telemonitoring as a substitute rather than additional care [20] or outsourcing technical difficulties experienced by patients to medical service centers [34].

This review showed that telemonitoring might shift health care utilization to outpatient settings, as opposed to only reducing inpatient admissions. This may complicate adoption, as the benefits may not be attributable to the same stakeholder as the costs. This is especially the case for health care systems where outpatient care is delivered by organizations other than inpatient care, such as that of Germany. In such cases, conflicting interests are to be expected, and health care payers have to come to an agreement with health care providers to overcome these issues [53]. Health care regulators can facilitate this process by creating and supporting new payment models such as shared savings and lump-sum payment models. 
Finally, the high costs of developing telemonitoring programs for a selected population can diminish and even cancel future monetary gains of reduced health care consumption [23]. One way of suppressing the costs of telemonitoring can be achieved through the use and development on a larger scale. Villani et al [23] showed that developing a telemonitoring system for 40 patients was not financially viable. In contrast, Vestergaard et al [46] reduced the costs of the program per patient by targeting a larger population, namely a whole region of North Denmark constituting 6700 patients. Health care providers should either use the scale realized by a third party or codevelop telemonitoring systems with other health care providers to realize possible scale advantages. In addition, in general, substitutive programs are expected to achieve higher financial savings.

\section{Future Research}

Future studies should consider clinical diversity by including subgroup meta-analyses or performing meta-regression, as opposed to pooled meta-analyses [54]. As mentioned previously, we found a high degree of clinical and methodological diversity [54]. Despite this, some meta-analyses [48,55] have found a low to moderate effect for certain health care utilization measures. The presence of high clinical diversity and low statistical heterogeneity may be due to minimal marginal effects of components in the telemonitoring program; a part of the intervention did not affect the outcome measure. If this is the case, certain (combinations of) telemonitoring components do not add value to the intervention. Yun et al [56] and Kotb et al [57] performed such analyses on all-cause mortality and hospitalization in patients with CHF. Certain program characteristics, such as having 3 or more physiological parameters [56] or including an electrocardiograph [57], were statistically associated with a reduction in all-cause mortality and hospitalization, respectively. Similarly, essential and effective parts of telemonitoring for reducing other health care utilization can be identified, thereby supporting the development of (cost-) effective telemonitoring programs. Clear descriptions of the intervention and context are needed to perform such analyses. This review can be used as guidance for forming subgroups or variables of interest for meta-regressions. As there are many more factors that may affect the impact of telemonitoring on health care utilization, qualitative research may be used to develop hypotheses and guide meta-regression protocols.

\section{Conclusions}

This review investigated the effects of telemonitoring programs on different aspects of health care utilization. Telemonitoring has the potential to reduce hospitalization rates. However, this was not achieved in most studies, as the number of non-ED visits increased in the majority of studies. The effect of telemonitoring on health care costs is highly ambiguous and depends on the effectiveness of the intervention in reducing health care utilization as well as on the costs of the telemonitoring program itself. Health care providers and payers should be aware that the majority of current telemonitoring programs do not result in a reduction in health care utilization and may even increase health care utilization by increasing the number of non-ED visits. Possible payer strategies should be focused at increasing the scale to reduce program costs and implement telemonitoring as a substitute to reduce possible increases in outpatient visits. Nevertheless, more focus is needed to determine the essential factors of telemonitoring programs that reduce health care utilization.

\section{Conflicts of Interest}

None declared.

\section{Multimedia Appendix 1}

Search queries for PubMed, Web of Science, and Embase.

[PDF File (Adobe PDF File), 67 KB-Multimedia Appendix 1]

\section{Multimedia Appendix 2}

Detailed characteristics of telemonitoring programs.

[PDF File (Adobe PDF File), 62 KB-Multimedia Appendix 2]

\section{Multimedia Appendix 3}

Risk of bias assessment: Cochrane Risk of Bias tool for randomized trials.

[PDF File (Adobe PDF File), 55 KB-Multimedia Appendix 3]

\section{Multimedia Appendix 4}

Risk of bias assessment: Risk of Bias in Non-randomized Studies of Interventions tool for non-randomized studies. [PDF File (Adobe PDF File), 117 KB-Multimedia Appendix 4]

\section{Multimedia Appendix 5}

Costs of telemonitoring programs. 
[PDF File (Adobe PDF File), 62 KB-Multimedia Appendix 5]

\section{References}

1. Berry C, Murdoch DR, McMurray JJ. Economics of chronic heart failure. Eur J Heart Fail 2001 Jun;3(3):283-291. [Medline: $\underline{11377998]}$

2. Cook C, Cole G, Asaria P, Jabbour R, Francis DP. The annual global economic burden of heart failure. Int J Cardiol 2014 Feb 15;171(3):368-376. [doi: 10.1016/j.ijcard.2013.12.028] [Medline: 24398230]

3. Wammes JJ, Auener S, van der Wees PJ, Tanke MA, Bellersen L, Westert GP, et al. Characteristics and health care utilization among patients with chronic heart failure: a longitudinal claim database analysis. ESC Heart Fail 2019 Dec;6(6):1243-1251 [FREE Full text] [doi: 10.1002/ehf2.12512] [Medline: 31556246]

4. White-Williams C, Unruh L, Ward K. Hospital utilization after a telemonitoring program: a pilot study. Home Health Care Serv Q 2015;34(1):1-13. [doi: 10.1080/01621424.2014.995256] [Medline: 25517540]

5. Gensini GF, Alderighi C, Rasoini R, Mazzanti M, Casolo G. Value of telemonitoring and telemedicine in heart failure management. Card Fail Rev 2017 Nov;3(2):116-121 [FREE Full text] [doi: 10.15420/cfr.2017:6:2] [Medline: 29387464]

6. Chaudhry SI, Phillips CO, Stewart SS, Riegel B, Mattera JA, Jerant AF, et al. Telemonitoring for patients with chronic heart failure: a systematic review. J Card Fail 2007 Feb;13(1):56-62 [FREE Full text] [doi: 10.1016/j.cardfail.2006.09.001] [Medline: 17339004]

7. Kulshreshtha A, Kvedar JC, Goyal A, Halpern EF, Watson AJ. Use of remote monitoring to improve outcomes in patients with heart failure: a pilot trial. Int J Telemed Appl 2010;2010:870959 [FREE Full text] [doi: 10.1155/2010/870959] [Medline: 20508741]

8. Burdese E, Testa M, Raucci P, Ferreri C, Giovannini G, Lombardo E, et al. Usefulness of a telemedicine program in refractory older congestive heart failure patients. Diseases 2018 Jan 20;6(1):10 [FREE Full text] [doi: 10.3390/diseases6010010] [Medline: 29361704]

9. Martín-Lesende I, Orruño E, Cairo C, Bilbao A, Asua J, Romo MI, et al. Assessment of a primary care-based telemonitoring intervention for home care patients with heart failure and chronic lung disease. The TELBIL study. BMC Health Serv Res 2011 Mar 08;11:56 [FREE Full text] [doi: 10.1186/1472-6963-11-56] [Medline: 21385401]

10. Inglis SC, Clark RA, Dierckx R, Prieto-Merino D, Cleland JGF. Structured telephone support or non-invasive telemonitoring for patients with heart failure. Cochrane Database Syst Rev 2015(10):CD007228. [doi: 10.1002/14651858.CD007228.pub3] [Medline: 26517969]

11. Bashi N, Karunanithi M, Fatehi F, Ding H, Walters D. Remote monitoring of patients with heart failure: an overview of systematic reviews. J Med Internet Res 2017 Jan 20;19(1):e18 [FREE Full text] [doi: 10.2196/jmir.6571] [Medline: 28108430]

12. Ligthelm RJ, Borzì V, Gumprecht J, Kawamori R, Wenying Y, Valensi P. Importance of observational studies in clinical practice. Clin Ther 2007 Jun;29(6 Pt 1):1284-1292. [doi: 10.1016/j.clinthera.2007.07.004] [Medline: 18036390]

13. Sinnige J, Korevaar JC, Westert GP, Spreeuwenberg P, Schellevis FG, Braspenning JC. Multimorbidity patterns in a primary care population aged 55 years and over. Fam Pract 2015 Oct;32(5):505-513 [FREE Full text] [doi: 10.1093/fampra/cmv037] [Medline: 26040310]

14. Bramer W, Bain P. Updating search strategies for systematic reviews using EndNote. J Med Libr Assoc 2017 Jul;105(3):285-289 [FREE Full text] [doi: 10.5195/jmla.2017.183] [Medline: 28670219]

15. Bramer WM, Giustini D, de Jonge GB, Holland L, Bekhuis T. De-duplication of database search results for systematic reviews in EndNote. J Med Libr Assoc 2016 Jul;104(3):240-243 [FREE Full text] [doi: 10.3163/1536-5050.104.3.014] [Medline: 27366130]

16. Field MJ. Telemedicine: a guide to assessing telecommunications in healthcare. J Digit Imaging 1997 Aug;10(3 Suppl 1):28 [FREE Full text] [doi: 10.1007/BF03168648] [Medline: 9268830]

17. Faragli A, Abawi D, Quinn C, Cvetkovic M, Schlabs T, Tahirovic E, et al. The role of non-invasive devices for the telemonitoring of heart failure patients. Heart Fail Rev 2020 Apr 27:1063-1080. [doi: 10.1007/s10741-020-09963-7] [Medline: 32338334]

18. Higgins JPT, Altman DG, Gøtzsche PC, Jüni P, Moher D, Oxman AD, et al. The Cochrane Collaboration's tool for assessing risk of bias in randomised trials. Br Med J 2011;343:d5928 [FREE Full text] [Medline: 22008217]

19. Sterne JA, Hernán MA, Reeves BC, Savović J, Berkman ND, Viswanathan M, et al. ROBINS-I: a tool for assessing risk of bias in non-randomised studies of interventions. Br Med J 2016 Oct 12;355:i4919 [FREE Full text] [doi: 10.1136/bmj.i4919] [Medline: 27733354]

20. Kraai I, de Vries A, Vermeulen K, van Deursen V, van der Wal M, de Jong R, et al. The value of telemonitoring and ICT-guided disease management in heart failure: Results from the IN TOUCH study. Int J Med Inform 2016 Jan;85(1):53-60 [FREE Full text] [doi: 10.1016/j.ijmedinf.2015.10.001] [Medline: 26514079]

21. Olivari Z, Giacomelli S, Gubian L, Mancin S, Visentin E, Di Francesco V, et al. The effectiveness of remote monitoring of elderly patients after hospitalisation for heart failure: the renewing health European project. Int J Cardiol 2018 Apr 15;257:137-142. [doi: 10.1016/j.ijcard.2017.10.099] [Medline: 29506685] 
22. Seto E, Leonard KJ, Cafazzo JA, Barnsley J, Masino C, Ross HJ. Mobile phone-based telemonitoring for heart failure management: a randomized controlled trial. J Med Internet Res 2012;14(1):e31 [FREE Full text] [doi: 10.2196/jmir.1909] [Medline: 22356799]

23. Villani A, Malfatto G, Compare A, Della RF, Bellardita L, Branzi G, et al. Clinical and psychological telemonitoring and telecare of high risk heart failure patients. J Telemed Telecare 2014 Dec;20(8):468-475. [doi: 10.1177/1357633X14555644] [Medline: 25339632]

24. Amir O, Ben-Gal T, Weinstein JM, Schliamser J, Burkhoff D, Abbo A, et al. Evaluation of remote dielectric sensing (ReDS) technology-guided therapy for decreasing heart failure re-hospitalizations. Int J Cardiol 2017 Aug 01;240:279-284 [FREE Full text] [doi: 10.1016/j.ijcard.2017.02.120] [Medline: 28341372]

25. Kotooka N, Kitakaze M, Nagashima K, Asaka M, Kinugasa Y, Nochioka K, HOMES-HF study investigators. The first multicenter, randomized, controlled trial of home telemonitoring for Japanese patients with heart failure: home telemonitoring study for patients with heart failure (HOMES-HF). Heart Vessels 2018 Aug;33(8):866-876. [doi: 10.1007/s00380-018-1133-5] [Medline: 29450689]

26. Domingo M, Lupón J, González B, Crespo E, López R, Ramos A, et al. [Noninvasive remote telemonitoring for ambulatory patients with heart failure: effect on number of hospitalizations, days in hospital, and quality of life. CARME (CAtalan Remote Management Evaluation) study]. Rev Esp Cardiol 2011 Apr;64(4):277-285 [FREE Full text] [doi: 10.1016/j.recesp.2010.10.032] [Medline: 21411210]

27. Hoban MB, Fedor M, Reeder S, Chernick M. The effect of telemonitoring at home on quality of life and self-care behaviors of patients with heart failure. Home Healthc Nurse 2013;31(7):368-377. [doi: 10.1097/NHH.0b013e318291fd56] [Medline: 23632274]

28. Koulaouzidis G, Barrett D, Mohee K, Clark AL. Telemonitoring in subjects with newly diagnosed heart failure with reduced ejection fraction: From clinical research to everyday practice. J Telemed Telecare 2018 Jan 01:1357633X17751004. [doi: 10.1177/1357633X17751004] [Medline: 29419343]

29. Lyngå P, Persson H, Hägg-Martinell A, Hägglund E, Hagerman I, Langius-Eklöf A, et al. Weight monitoring in patients with severe heart failure (WISH). A randomized controlled trial. Eur J Heart Fail 2012 Apr;14(4):438-444 [FREE Full text] [doi: 10.1093/eurjhf/hfs023] [Medline: 22371525]

30. Maeng DD, Starr AE, Tomcavage JF, Sciandra J, Salek D, Griffith D. Can telemonitoring reduce hospitalization and cost of care? A health plan's experience in managing patients with heart failure. Popul Health Manag 2014 Dec;17(6):340-344. [doi: 10.1089/pop.2013.0107] [Medline: 24865986]

31. Pedone C, Rossi FF, Cecere A, Costanzo L, Antonelli IR. Efficacy of a physician-led multiparametric telemonitoring system in very old adults with heart failure. J Am Geriatr Soc 2015 Jun;63(6):1175-1180. [doi: 10.1111/jgs.13432] [Medline: 26031737]

32. Soran OZ, Feldman AM, Piña IL, Lamas GA, Kelsey SF, Selzer F, et al. Cost of medical services in older patients with heart failure: those receiving enhanced monitoring using a computer-based telephonic monitoring system compared with those in usual care: the Heart Failure Home Care trial. J Card Fail 2010 Nov;16(11):859-866. [doi: 10.1016/j.cardfail.2010.05.028] [Medline: 21055649]

33. Tompkins C, Orwat J. A randomized trial of telemonitoring heart failure patients. J Healthc Manag 2010;55(5):312-22; discussion 322. [Medline: 21077581]

34. van der Burg JM, Aziz NA, Kaptein MC, Breteler MJ, Janssen JH, van Vliet L, et al. Long-term effects of telemonitoring on healthcare usage in patients with heart failure or COPD. Clinical eHealth 2020;3:40-48. [doi: 10.1016/j.ceh.2020.05.001]

35. Veenstra W, Op den Buijs J, Pauws S, Westerterp M, Nagelsmit M. Clinical effects of an optimised care program with telehealth in heart failure patients in a community hospital in the Netherlands. Neth Heart J 2015 Jun;23(6):334-340 [FREE Full text] [doi: 10.1007/s12471-015-0692-7] [Medline: 25947078]

36. Ware P, Ross HJ, Cafazzo JA, Boodoo C, Munnery M, Seto E. Outcomes of a heart failure telemonitoring program implemented as the standard of care in an outpatient heart function clinic: pretest-posttest pragmatic study. J Med Internet Res 2020 Feb 08;22(2):e16538 [FREE Full text] [doi: 10.2196/16538] [Medline: 32027309]

37. Williams C, Wan TT. A cost analysis of remote monitoring in a heart failure program. Home Health Care Serv Q 2016;35(3-4):112-122. [doi: 10.1080/01621424.2016.1227009] [Medline: 27552654]

38. Zan S, Agboola S, Moore SA, Parks KA, Kvedar JC, Jethwani K. Patient engagement with a mobile web-based telemonitoring system for heart failure self-management: a pilot study. JMIR Mhealth Uhealth 2015;3(2):e33 [FREE Full text] [doi: 10.2196/mhealth.3789] [Medline: 25842282]

39. Frederix I, Vanderlinden L, Verboven A, Welten M, Wouters D, De Keulenaer G, et al. Long-term impact of a six-month telemedical care programme on mortality, heart failure readmissions and healthcare costs in patients with chronic heart failure. J Telemed Telecare 2019 Jun;25(5):286-293. [doi: 10.1177/1357633X18774632] [Medline: 29742959]

40. Park C, Otobo E, Ullman J, Rogers J, Fasihuddin F, Garg S, et al. Impact on readmission reduction among heart failure patients using digital health monitoring: feasibility and adoptability study. JMIR Med Inform 2019 Nov 15;7(4):e13353 [FREE Full text] [doi: 10.2196/13353] [Medline: $\underline{\text { 31730039] }}$ 
41. Riley WT, Keberlein P, Sorenson G, Mohler S, Tye B, Ramirez AS, et al. Program evaluation of remote heart failure monitoring: healthcare utilization analysis in a rural regional medical center. Telemed J E Health 2015 Mar;21(3):157-162 [FREE Full text] [doi: 10.1089/tmj.2014.0093] [Medline: 25025239]

42. Bakhshi S, Li X, Semenov N, Apodaca-Madrid J, Mahoor MH, Newman KE, et al. Congestive heart failure home monitoring pilot study in urban Denver. Annu Int Conf IEEE Eng Med Biol Soc 2011;2011:3150-3153. [doi: 10.1109/IEMBS.2011.6090859] [Medline: 22255008]

43. Delaney C, Apostolidis B, Bartos S, Morrison H, Smith L, Fortinsky R. A randomized trial of telemonitoring and self-care education in heart failure patients following home care discharge. Home Health Care Manag Pract 2013 Feb 20;25(5):187-195. [doi: $10.1177 / 1084822312475137]$

44. Dendale P, De Keulenaer G, Troisfontaines P, Weytjens C, Mullens W, Elegeert I, et al. Effect of a telemonitoring-facilitated collaboration between general practitioner and heart failure clinic on mortality and rehospitalization rates in severe heart failure: the TEMA-HF 1 (TElemonitoring in the MAnagement of Heart Failure) study. Eur J Heart Fail 2012 Mar;14(3):333-340 [FREE Full text] [doi: 10.1093/eurjhf/hfr144] [Medline: 22045925]

45. Eilat-Tsanani S, Golovner M, Marcus O, Dayan M, Sade Z, Iktelat A, et al. Evaluation of telehealth service for patients with congestive heart failure in the north of Israel. Eur J Cardiovasc Nurs 2016 Apr;15(3):e78-e84. [doi: 10.1177/1474515115602677] [Medline: 26311654]

46. Vestergaard AS, Hansen L, Sørensen SS, Jensen MB, Ehlers LH. Is telehealthcare for heart failure patients cost-effective? An economic evaluation alongside the Danish TeleCare North heart failure trial. BMJ Open 2020 Jan 27;10(1):e031670 [FREE Full text] [doi: 10.1136/bmjopen-2019-031670] [Medline: 31992604$]$

47. Vuorinen A, Leppänen J, Kaijanranta H, Kulju M, Heliö T, van Gils M, et al. Use of home telemonitoring to support multidisciplinary care of heart failure patients in Finland: randomized controlled trial. J Med Internet Res 2014;16(12):e282 [FREE Full text] [doi: 10.2196/jmir.3651] [Medline: 25498992]

48. Pekmezaris R, Tortez L, Williams M, Patel V, Makaryus A, Zeltser R, et al. Home telemonitoring in heart failure: a systematic review and meta-analysis. Health Aff (Millwood) 2018 Dec;37(12):1983-1989. [doi: 10.1377/hlthaff.2018.05087] [Medline: 30633680]

49. Polisena J, Tran K, Cimon K, Hutton B, McGill S, Palmer K, et al. Home telemonitoring for congestive heart failure: a systematic review and meta-analysis. J Telemed Telecare 2010;16(2):68-76. [doi: 10.1258/jtt.2009.090406] [Medline: 20008054]

50. Dang S, Dimmick S, Kelkar G. Evaluating the evidence base for the use of home telehealth remote monitoring in elderly with heart failure. Telemed J E Health 2009 Oct;15(8):783-796. [doi: 10.1089/tmj.2009.0028] [Medline: 19831704]

51. Cleland JG, Louis AA, Rigby AS, Janssens U, Balk AH, TEN- H. Noninvasive home telemonitoring for patients with heart failure at high risk of recurrent admission and death: the Trans-European Network-Home-Care Management System (TEN-HMS) study. J Am Coll Cardiol 2005 May 17;45(10):1654-1664 [FREE Full text] [doi: 10.1016/j.jacc.2005.01.050] [Medline: 15893183]

52. Brons M, Koudstaal S, Asselbergs FW. Algorithms used in telemonitoring programmes for patients with chronic heart failure: a systematic review. Eur J Cardiovasc Nurs 2018 Oct;17(7):580-588 [FREE Full text] [doi: 10.1177/1474515118786838] [Medline: 29954184]

53. Desveaux L, Soobiah C, Bhatia RS, Shaw J. Identifying and overcoming policy-level barriers to the implementation of digital health innovation: qualitative study. J Med Internet Res 2019 Dec 20;21(12):e14994 [FREE Full text] [doi: 10.2196/14994] [Medline: 31859679]

54. Deeks J, Higgins J, Altman D. Chapter 10: Analysing data and undertaking meta-analyses. In: Cochrane Handbook for Systematic Reviews of Interventions. Chichester (UK): John Wiley \& Sons; 2019.

55. Klersy C, De Silvestri A, Gabutti G, Regoli F, Auricchio A. A meta-analysis of remote monitoring of heart failure patients. J Am Coll Cardiol 2009 Oct 27;54(18):1683-1694 [FREE Full text] [doi: 10.1016/j.jacc.2009.08.017] [Medline: 19850208]

56. Yun JE, Park J, Park H, Lee H, Park D. Comparative effectiveness of telemonitoring versus usual care for heart failure: a systematic review and meta-analysis. J Card Fail 2018 Jan;24(1):19-28. [doi: 10.1016/j.cardfail.2017.09.006] [Medline: $\underline{28939459]}$

57. Kotb A, Cameron C, Hsieh S, Wells G. Comparative effectiveness of different forms of telemedicine for individuals with heart failure (HF): a systematic review and network meta-analysis. PLoS One 2015;10(2):e0118681 [FREE Full text] [doi: 10.1371/journal.pone.0118681] [Medline: 25714962]

\section{Abbreviations}

CHF: chronic heart failure

ED: emergency department

NYHA: New York Heart Association

RCT: randomized controlled trial

STS: structured telephone support 
Edited by R Kukafka; submitted 23.12.20; peer-reviewed by T Wan, W Bramer, A Beleigoli; comments to author 04.03.21; revised version received 18.03.21; accepted 30.06.21; published 29.09.21

Please cite as:

Auener SL, Remers TEP, van Dulmen SA, Westert GP, Kool RB, Jeurissen PPT

The Effect of Noninvasive Telemonitoring for Chronic Heart Failure on Health Care Utilization: Systematic Review

$J$ Med Internet Res 2021;23(9):e26744

URL: https://www.jmir.org/2021/9/e26744

doi: 10.2196/26744

PMID:

(CStefan L Auener, Toine E P Remers, Simone A van Dulmen, Gert P Westert, Rudolf B Kool, Patrick P T Jeurissen. Originally published in the Journal of Medical Internet Research (https://www.jmir.org), 29.09.2021. This is an open-access article distributed under the terms of the Creative Commons Attribution License (https://creativecommons.org/licenses/by/4.0/), which permits unrestricted use, distribution, and reproduction in any medium, provided the original work, first published in the Journal of Medical Internet Research, is properly cited. The complete bibliographic information, a link to the original publication on https://www.jmir.org/, as well as this copyright and license information must be included. 